\section{Continuous intravenous milrinone therapy at home}

Long-term milrinone therapy is common in patients with advanced heart failure awaiting transplant, although there is evidence that long-term administration of inotropic therapy increases mortality in these patients. Since the wait for a donor heart is often lengthy, Brozena et al. have studied the feasibility and safety of continuous intravenous milrinone therapy administered at home.

Sixty hospitalized patients listed as Status 1B for heart transplantion were included in the trial. All were inotrope-dependent and had an ICD. After initiation and adjustment of milrinone dose during hospitalization, milrinone was administered at home via an intravenous catheter and a preset, computerized pump.

During the 43-month follow-up period, 53 $(88.3 \%)$ patients underwent successful heart transplantation. Two patients died while waiting for transplantation, two were removed from the list, and the remaining three patients were awaiting transplantation at the end of the study. As expected, hospitalization was frequent: there were 89 admissions for 46 patients, most of which were for heart failure. Six patients had ICD firings for ventricular tachycardia. Quality of life 1 month after discharge was significantly improved compared with baseline. Although cost data were unavailable, the estimated cost of home care was only $4.2 \%$ of the average daily charge of remaining hospitalized.

In conclusion, home administration of intravenous milrinone therapy was a safe alternative to extended hospitalization in these inotropedependent patients awaiting transplant. The authors note that rehospitalization is probable and they recommend that an ICD should be implanted.

Original article Brozena SC et al. (2004) A prospective study of continuous intravenous milrinone therapy for Status IB patients awaiting heart transplant at home. J Heart Lung Transplant 23: 1082-1086

\section{Hospital-based discharge medication program improves outcomes}

Despite a wealth of evidence supporting the use of medical therapies in the secondary prevention of cardiovascular disorders, many patients are discharged from hospital without the appropriate medications. Lappé and colleagues have attempted to bridge this treatment gap by designing a 'discharge medication program' (DMP).

This quality-improvement initiative, implemented in 1999, covered 10 hospitals of Intermountain Health Care in the US. The aim was to ensure the appropriate prescription of evidence-based medications (aspirin, statins, $\beta$-blockers, ACE inhibitors and warfarin) at discharge. To this end, the patient discharge form was amended to show the indications for each medication. Physicians were required to check the appropriate box on the form, or to note any contraindication. Tracking of discharge medication data began the year before implementation of the DMP and is ongoing.

One year after the DMP began, prescription rates for each of the targeted medications had increased significantly, reaching $90 \%$ of appropriate patients. At 30 days' follow-up, the relative risk of death and readmission was significantly lower for patients in the DMP period $(n=31,465)$ than for those admitted before the DMP was implemented $(n=26,000)$. The risk of death continued to decrease significantly in the DMP group at 1 year, although the risk of readmission remained stable.

Lappé et al. conclude that the DMP was feasible and sustainable in this multihospital setting. Importantly, this approach might improve long-term cardiovascular readmission rates and mortality at low cost.

Original article Lappé JM et al. (2004) Improvements in 1-year cardiovascular clinical outcomes associated with a hospital-based discharge medication program. Ann Intern Med 141: 446-453

\section{GLOSSARY}

ICD

Implantable cardioverterdefibrillator

ACE

Angiotensin-converting enzyme 